\title{
Risk factors and effective management of preeclampsia
}

\author{
This article was published in the following Dove Press journal: \\ Integrated Blood Pressure Control \\ 3 March 2015 \\ Number of times this article has been viewed
}

\author{
Fred A English' \\ Louise C Kenny' \\ Fergus P McCarthy ${ }^{1,2}$ \\ 'Irish Centre for Fetal and Neonatal \\ Translational Research (INFANT), \\ Department of Obstetrics and \\ Gynaecology, University College \\ Cork, Cork, Ireland; 'Women's \\ Health Academic Centre, King's \\ Health Partners, St Thomas' Hospital, \\ London, UK
}

\begin{abstract}
Preeclampsia, a hypertensive disorder of pregnancy is estimated to complicate $2 \%-8 \%$ of pregnancies and remains a principal cause of maternal and fetal morbidity and mortality. Preeclampsia may present at any gestation but is more commonly encountered in the third trimester. Multiple risk factors have been documented, including: family history, nulliparity, egg donation, diabetes, and obesity. Significant progress has been made in developing tests to predict risk of preeclampsia in pregnancy, but these remain confined to clinical trial settings and center around measuring angiogenic profiles, including placental growth factor or newer tests involving metabolomics. Less progress has been made in developing new treatments and therapeutic targets, and aspirin remains one of the few agents shown to consistently reduce the risk of developing preeclampsia. This review serves to discuss recent advances in risk factor identification, prediction techniques, and management of preeclampsia in antenatal, intrapartum, and postnatal patients.
\end{abstract}

Keywords: pregnancy, treatment, risk reduction, prediction

\section{Introduction}

The International Society for the Study of Hypertension in Pregnancy defines preeclampsia as hypertension of at least $140 / 90 \mathrm{mmHg}$ on two separate occasions $\geq 4$ hours apart accompanied by significant proteinuria of at least $0.3 \mathrm{~g}$ in a 24-hour collection of urine (or $>30 \mathrm{mg} / \mathrm{mmol}$ protein/creatinine ratio), arising de novo after the 20th week of gestation in a previously normotensive woman and resolving completely by the 6th postpartum week. ${ }^{1,2}$ Preeclampsia complicates $2 \%-8 \%$ of pregnancies and occurs most commonly during the second half of pregnancy. ${ }^{2}$ While overall rates of preeclampsia remain static, rates of severe preeclampsia appear to have increased over recent decades. ${ }^{3}$ Preeclampsia is responsible for approximately $15 \%$ of all direct maternal deaths in the UK. Furthermore, the condition increases perinatal mortality, largely through iatrogenic prematurity, by five-fold. ${ }^{4-7}$

Recent reports from the World Health Organization (WHO) estimate that preeclampsia is directly responsible for 70,000 maternal deaths annually worldwide. ${ }^{8}$ In addition to the maternal mortality and morbidity, preeclampsia accounts for 500,000 infant deaths annually. ${ }^{4}$ Preeclampsia is a heterogeneous disorder affecting multiple organ systems. While the severity of clinical presentation is highly variable, outcomes are usually favorable when mild preeclampsia develops after the 36th week. The risk of adverse maternal and perinatal outcome increases significantly when preeclampsia develops early, before 33 weeks' gestation, or at any gestation in those with preexisting medical conditions. Outcomes are less favorable in women living in developing
Correspondence: Fred A English INFANT, Cork University Maternity Hospital, Cork, Ireland

Tel +353870553639

Email fred@umail.ucc.ie 
countries, regardless of gestation or severity of clinical presentation. ${ }^{9}$

Preeclampsia results from impaired trophoblast differentiation and invasion in early pregnancy, which stimulates sustained oxidative stress and a systemic inflammatory response. ${ }^{10}$ Recent work has suggested that the pathology might be better viewed as resulting from extrinsic and intrinsic factors. Abnormal placentation has been suggested as an extrinsic cause leading to early onset of preeclampsia. Recent work has suggested that late onset preeclampsia ( $\geq 34$ weeks' gestation) may be triggered by a distinct, intrinsic pathology involving microvillus overcrowding. This is thought to occur as placental growth reaches its limits at term, with diminishing villous pore size impeding perfusion and increasing oxidative stress. This theory extends to suggest that oxidative stress proteins modulate the maternal response to developing preeclampsia through regulation of various growth factors. ${ }^{11}$

It is widely accepted that all etiological processes are compounded by maternal and fetal genetic composition and environmental factors. Less well understood is how the interplay of these factors influences the degree of systemic endothelial dysfunction and maternal hypertension. ${ }^{12}$ This is supported by evidence demonstrating impaired placental perfusion in women who had growth-restricted babies in the absence of preeclampsia and the fact that $70 \%$ of babies born to women with preeclampsia are not growth restricted. ${ }^{13,14}$ These differences may be explained by maternal risk factors adversely affecting the ability of the placenta to cope with reduced placental perfusion, with a resultant reduction in the supply of nutrients to the fetus. In addition, it is widely accepted that systemic inflammation plays an important role in the development of this syndrome as evidenced by exaggerated leukocytosis, extensive platelet activation, and enhanced complement activation in preeclampsia. ${ }^{12,15,16}$

\section{Risk factors for preeclampsia}

Early identification of preeclampsia (and if possible, prevention) is a core tenet of adequate management. The National Institute for Health and Care Excellence (NICE) recommends that women at high risk of preeclampsia be identified before week 13 of gestation and low-dose aspirin commenced until 36 weeks' gestation. There are many conditions and health risk behaviors that are thought to predispose to preeclampsia; these are summarized in Table 1. High-risk women include those with preexisting hypertension, chronic kidney disease, insulin-dependent diabetics, and women with previous early onset preeclampsia. ${ }^{17}$ Administration of low-dose aspirin
Table I Summary of principal risk factors for preeclampsia

\begin{tabular}{ll}
\hline Risk factor & Mean RR (95\% Cl) \\
\hline Antiphospholipid syndrome & $9.72(4.34-21.75)$ \\
Relative risk of preeclampsia & $7.19(5.85-8.83)$ \\
Previous preeclampsia & $7.19(5.85-8.83)$ \\
Insulin-dependent diabetes & $3.56(2.54-4.99)$ \\
Multiple pregnancy & $2.93(2.04-4.2 \mathrm{I})$ \\
Nulliparity & $2.9 \mathrm{I}(\mathrm{I} .28-6.6 \mathrm{I})$ \\
Family history of preeclampsia & $2.90(\mathrm{I} .70-4.93)$ \\
Obesity & $2.47(\mathrm{I} .66-3.67)$ \\
Age $>40$ years & $1.96(\mathrm{I} .34-2.87)$ \\
Preexisting hypertension & $\mathrm{I} .38(\mathrm{I} .0 \mathrm{I}-\mathrm{I} .87)$ \\
\hline
\end{tabular}

Abbreviations: $\mathrm{Cl}$, confidence interval; $\mathrm{RR}$, relative risk.

to women at moderate-to-high risk has been shown to be beneficial and reduces the incidence of preeclampsia by approximately $15 \% .{ }^{18}$ Preeclampsia is more common in primigravida women and the risk of preeclampsia increases the greater the interval between pregnancies. ${ }^{19}$ Age greater than 40 years of age increases risk (relative risk [RR]: 1.96, 95\% confidence interval [CI]: 1.34-2.87), as does a previous history of preeclampsia (RR: 7.19, 95\% CI: 5.85-8.83), pre-pregnancy obesity (RR: $2.47,95 \%$ CI: $1.66-3.67$ ), and women who become pregnant with donor eggs, embryo donation, or donor insemination. ${ }^{9}$ Other risk factors include diabetes (RR: 3.56, 95\% CI: 2.54-4.99), preexisting hypertension (RR: $1.38,95 \%$ CI: $1.01-1.87$ ), those with a family history of preeclampsia (RR: $2.90,95 \%$ CI: 1.70-4.93), and women suffering from medical conditions such as antiphospholipid syndrome (RR: 9.72, 95\% CI: 4.34-21.75). In addition, it appears that various paternal factors can increase the risk of a pregnancy being complicated by preeclampsia. ${ }^{9,20}$

Preeclampsia is in itself a risk factor for both early and late complications affecting mother and baby. Preeclampsia may be complicated by seizures (eclampsia; $2-3$ vs $40-90$ cases/ 10,000 births in Europe and developing countries, respectively), pulmonary edema, placental abruption $(1 \%-4 \%)$, oligohydramnios, and fetal growth restriction (up to $30 \%$ ). ${ }^{21}$ Long-term effects on offspring include an increased risk of stroke and hypertension. ${ }^{21-23}$ In addition to the long-term health implications for offspring, women with preeclampsia have 3.7 times higher risk of developing hypertension later in life, 2.2 times increased risk of coronary heart disease, and 1.8 times higher risk of stroke. ${ }^{24}$

\section{Prediction}

Management of preeclampsia centers on early recognition and timely delivery to prevent serious morbidity and mortality. Despite recent advances in our understanding of the etiology of preeclampsia, there is still no clinically 
useful screening test. The diagnosis of preeclampsia in the community is by detection of persistent hypertension and proteinuria, which then warrants referral to tertiary units for further management. ${ }^{25}$ However, recent research has identified novel predictive and diagnostic tests.

Much research has focused on the angiogenic factors, including the pro-angiogenic factors vascular endothelial growth factor (VEGF) and placental growth factor (P1GF), in addition to the two anti-angiogenic proteins, soluble endoglin (sEng) and sFlt-1. ${ }^{26-28}$ PlGF, a member of the VEGF family, is an angiogenic, proinflammatory factor produced by trophoblast cells, and has a central role in the regulation of VEGF-dependent angiogenesis, has been long associated with the pathogenesis of preeclampsia, and is thought to be a secondary marker for the placental dysfunction that occurs in preeclampsia. ${ }^{29,30}$ Recent investigations have examined the clinical relevance of low plasma PlGF concentration $(<5$ th centile for gestation, Alere ${ }^{\mathrm{TM}}$ Triage assay) in women in whom there is a clinical suspicion of preeclampsia at 20-35 weeks' gestation (and up to 41 weeks' gestation as a secondary analysis). Of 625 women enrolled in the study, PlGF $<5$ th centile had high sensitivity $(0.96$; 95\% CI: $0.89-0.99)$ and negative predictive value $(0.98 ; 95 \%$ CI: $0.93-0.99)$ for development of preeclampsia in the following 2 weeks; specificity was lower $(0.55 ; 95 \%$ CI: $0.48-0.61){ }^{27}$

Impedance to flow in the maternal uterine arteries normally decreases as pregnancy progresses. Increased impedance in the uterine arteries is an early radiographic feature of preeclampsia. This increased resistance likely reflects high downstream resistance due to defective differentiation of trophoblasts with resulting defective invasion of spiral arteries and failure of these vessels to transform into low-resistance vessels. Although Doppler ultrasound can predict women at increased risk of preeclampsia, it is a poor screening tool. In low-risk women, the risk of severe preeclampsia is best predicted by elevation of the pulsatility index in the second trimester (sensitivity: 78\%, specificity: 95\%). In women at high risk of preeclampsia, the risk of severe preeclampsia is best predicted by elevated resistance index in the second trimester (sensitivity: 80\%, specificity: 78\%). ${ }^{31,32}$

Metabolomics, the scientific study of chemical processes involving metabolites, the intermediates, and products of metabolism, is an emerging predictive technology. Metabolomic changes may be detected in early pregnancy thus allowing early intervention and effective management. Metabolomics and associated technologies have successfully detected metabolic signatures of preeclampsia in early pregnancy. A recent profiling study, utilizing multivariate predictive modeling combining 14 metabolites, gave an odds ratio for developing preeclampsia of 36 (95\% CI: 12-108), with an area under the receiver operator characteristic curve of $0.94 .{ }^{33}$ A Phase IIa clinical study is planned to further investigate these emerging metabolomic technologies. ${ }^{34}$ Another recent study has demonstrated characteristic proteomic biomarkers in the urine of women. It is thought that excretion of these factors may precede the development of symptoms and predict the likely severity of the disease. ${ }^{35}$

Predicting preeclampsia in healthy nulliparous women using risk factor analysis is not robust but may, with addition of other biochemical features, offer a solution to best identify those at risk. ${ }^{36}$ The heterogeneous nature of preeclampsia makes it unlikely that a single clinical risk factor or biomarker in early pregnancy will ever predict those women likely to develop preeclampsia. A recent study has combined phenotypical features with biomarkers to produce an algorithm that detects preeclampsia in a significant portion of first-time mothers. This study created a model for the prediction of preeclampsia, which included PlGF, mean arterial pressure, and body mass index at 14-16 weeks' gestation, the consumption of $\geq 3$ pieces of fruit per day, and mean uterine artery resistance index. ${ }^{37}$

\section{Management of preeclampsia}

The management of preeclampsia has not changed significantly over time, possibly as a result of the poor progress being made in our understanding of the condition. ${ }^{38}$ Effective management of preeclampsia may be divided into three categories; prevention of preeclampsia, early detection, and treatment. Women considered to be at high risk of preeclampsia (such as those with chronic hypertension, coexisting renal disease, or antiphospholipid syndrome should be referred for pre-pregnancy counseling to identify modifiable risk factors). This management may involve cessation of smoking advice, dietary advice, adjustment of medications to optimize medical conditions such as preexisting renal disease, and cessation of potentially teratogenic agents such as warfarin and angiotensin-converting enzyme (ACE) inhibitors. Baseline levels for blood pressure, platelet function, renal function (plasma creatinine and urinary protein/creatinine ratios), and liver function should be recorded. Optimal pre-pregnancy health may reduce risk of developing preeclampsia. Women thought to be at high risk should be given low-dose aspirin before 12 weeks' gestation until 36 weeks' gestation. ${ }^{17,39}$ Calcium supplementation ( $\geq 1 \mathrm{~g} /$ day) is associated with a significant reduction in the risk of preeclampsia, particularly for women with low-calcium diets. ${ }^{40}$ 
No ideal antenatal program of care has been identified for women at high risk of preeclampsia. However, these women are often seen more frequently antenatally and management includes an early accurate dating scan, anomaly scan, regular (4 weekly to weekly depending on risk factors and gestation) blood pressure and urine checks, and 4 weekly growth assessments to monitor for fetal growth restriction if preeclampsia is detected. As one of the risk factors for preeclampsia is nulliparity, often women have no preexisting comorbidities. As a result, regular antenatal check-ups are advocated to detect hypertension and proteinuria until better widespread predictive tests are available, which may help stratify women into high- and low-risk groups. It is unclear whether mild preeclampsia is best managed in hospital or as an outpatient and it is generally dependent on individual hospital policy.

General measures once severe preeclampsia has been diagnosed include admission and consideration for transfer to a high dependency care unit or delivery suite with multidisciplinary team input including obstetrics, anesthetics, hematology, and neonatology. Ideally, national or local protocols should be agreed upon and implemented to ensure optimal care. Similarly, any decision for pharmacological intervention should consider side effect profile, contraindication, and dosing considerations per local formulary guidelines. Steroids should be considered to reduce the risk of respiratory distress syndrome if delivery by cesarean section is planned or if the fetus is preterm.

Baseline investigations should include serum electrolytes (Na, K, urea, creatinine, and uric acid), liver function tests (monitoring in particular for raised transaminases), full blood count (monitoring for thrombocytopenia and hemolysis), clotting (especially if liver function tests are abnormal), and group and save serum. Urinary protein/creatinine ratio or 24-hour urine collection should be obtained to correctly diagnose preeclampsia and to demonstrate the degree of renal impairment along with serum urea and creatinine. All tests should be checked daily, or more frequently if abnormal and interpreted with reference to normal values in pregnancy. In cases of severe preeclampsia, blood pressure and pulse should be measured every 15 minutes until stabilized - then half hourly. Careful hourly fluid balances should be maintained to reduce the risk of fluid overload. If necessary, an indwelling catheter should be inserted. Vital signs should be measured at regular intervals; respiratory rate measured hourly and temperature measured every 4 hours. Fetal well-being should be assessed carefully with cardiotocography and growth scan, liquor assessment, and umbilical artery Doppler.
The exact level at which antihypertensive treatment is commenced is unclear but a blood pressure $\geq 160 / 110 \mathrm{mmHg}$ should be considered a medical emergency due to the risk of stroke. The aim of antihypertensive therapy is to reduce blood pressure to $<160 / 105 \mathrm{mmHg}$ (mean arterial pressure $<125 \mathrm{mmHg}$ ). Blood pressure may drop suddenly on commencement of treatment; therefore, dosage should be titrated gradually to avoid affecting uteroplacental circulation, which may result in fetal distress. Oral antihypertensives include methyldopa, labetalol, and calcium antagonists such as nifedipine. For acute treatment of hypertensive crises, intravenous medication may be needed. Again, local and national protocols should be in place and followed. The first antihypertensive agent of choice is labetalol, a mixed alpha/ beta adrenergic antagonist. Two doses can be administered orally. A period of 30 minutes should elapse prior to administration of a second oral dose. In the event that oral therapy fails to elicit a response or is poorly tolerated, proceed to give an intravenous bolus. A labetalol infusion should be commenced via syringe pump following the bolus dose if required. The principal contraindication for labetalol is severe asthma. The agent is cautioned in cardiac disease. ${ }^{38}$ The second antihypertensive agent of choice is hydralazine, a direct-acting smooth muscle relaxant. A bolus infusion can be given every 5 minutes with close monitoring of blood pressure. This may be followed by a basal infusion. All doses should be confirmed with updated formulary and pharmacy locally. Continuous fetal monitoring is appropriate in the management of severe preeclampsia.

Magnesium sulfate $\left(\mathrm{MgSO}_{4}\right)$ is the drug of choice for prevention of eclampsia. ${ }^{41}$ It is now accepted that other anticonvulsant agents such as diazepam are not appropriate treatments in the prevention of convulsions (eclampsia). A loading dose of $4 \mathrm{~g}$ of $\mathrm{MgSO}_{4}$ is given, followed by a maintenance infusion of $1 \mathrm{~g}$ /hour, generally for 24 hours after delivery. A documented medical assessment should occur at 4 hourly intervals and all patients should have continuous pulse oximetry, hourly assessment of urine output and respiratory rate, and reflexes checked every 4 hours. Cessation or reduction of magnesium therapy should occur if biceps reflex is absent or respiratory rate is $<12 /$ minute.

Adverse effects of $\mathrm{MgSO}_{4}$ toxicity include: paralysis, absence of reflexes, depression of respiratory drive, and arrhythmias. The corrective treatment for toxicity is $10 \mathrm{~mL}$ $10 \%$ calcium gluconate, which should be given gradually intravenously. Note that $97 \%$ of $\mathrm{MgSO}_{4}$ is cleared by the kidneys; therefore, oliguria ( $<80 \mathrm{~mL} / 24$ hours) can lead to toxicity. In the presence of oliguria, abnormal liver 
function tests, or further seizures, $\mathrm{MgSO}_{4}$ levels should be checked, aiming for a therapeutic range of $2-4 \mathrm{mmol} / \mathrm{L}$.

Fluid management should be closely monitored, as these patients have a reduced plasma volume and are at increased risk of pulmonary edema. Total input should not exceed $80 \mathrm{~mL} /$ hour (approximately $1 \mathrm{~mL} / \mathrm{kg} /$ hour). Oxytocin, if required, should be used at high concentrations and included as part of the total fluid input. Oliguria should not precipitate any individual intervention, save to encourage early delivery.

The only cure for preeclampsia is delivery, which should be considered only when the mother has been stabilized. The HYPITAT trial: a multicenter, open-label randomized controlled trial, randomized women with gestational hypertension or mild preeclampsia to induction of labor versus expectant monitoring after 36 weeks' gestation. ${ }^{42}$ They demonstrated that 117 (31\%) women who had labor induced suffered a poor maternal outcome compared with 166 (44\%) monitored expectantly (RR: $0.71,95 \%$ CI: $0.59-0.86$, $P<0.001)$. As a result, they advocate induction of labor beyond 37 weeks' gestation.

Delivery is indicated prior to this in the event of fetal distress, inability to control maternal blood pressure, eclampsia, worsening biochemistry, or worsening maternal symptoms. Delivery need not be by cesarean section, but if gestation $<32$ weeks, it is probably preferable. After 34 weeks of gestation, vaginal delivery can be considered. Vaginal prostaglandin increases the chance of success. Antihypertensive treatment should be continued throughout assessment and labor. Management of the third stage should utilize 5 IU of intravenous oxytocin, not ergometrine or oxytocin/ ergometrine, which may cause a hypertensive crisis.

Women who have had hypertension during pregnancy should be followed up postpartum to ensure full resolution of their hypertension. Women who have suffered from preeclampsia during their pregnancy should be advised that they are at increased risk of developing cardiovascular and renal disease later in life. Discharge should be followed by regular attendance with a general practitioner so that blood pressure can be closely monitored.

Those who have been diagnosed with severe preeclampsia are more likely to experience recurrence in their next pregnancy; however, the phenotype is typically less severe, with presentation approximately 2-3 weeks later in gestation. Women who have experienced severe early onset preeclampsia, especially if complicated by growth restriction or late fetal loss, should undergo testing for antiphospholipid syndrome. It may be necessary to discuss the implications of these results on future pregnancies. Similarly, those who have experienced severe preeclampsia complicated by stillbirth or abruption, may need to be tested for factor V Leiden, protein $\mathrm{S}$, protein $\mathrm{C}$, hyperhomocysteinuria, and antithrombin deficiency; however, the impact of these conditions on future pregnancies is less well understood.

Pre-conceptual counseling should be offered for all those with essential hypertension, as certain antihypertensive medications such as ACE inhibitors are teratogenic and may need to altered pre-conceptually.

The NICE provide graphical illustrations of guidelines for management of preeclampsia, which are suitable for use as reference in clinical settings. ${ }^{17}$ Physicians should utilize up-to-date guidelines from their jurisdiction in active management.

\section{Disclosure}

The authors report no conflicts of interest in this work.

\section{References}

1. Davey DA, MacGillivray I. The classification and definition of the hypertensive disorders of pregnancy. Am J Obstet Gynecol. 1988; 158(4):892-898.

2. Steegers EA, von Dadelszen P, Duvekot JJ, Pijnenborg R. Pre-eclampsia. Lancet. 2010;376(9741):631-644.

3. Ananth CV, Keyes KM, Wapner RJ. Pre-eclampsia rates in the United States, 1980-2010: age-period-cohort analysis. BMJ. 2013;347: f6564.

4. Khan KS, Wojdyla D, Say L, Gülmezoglu AM, Van Look PFA. WHO analysis of causes of maternal death: a systematic review. Lancet. 2006; 367(9516):1066-1074.

5. CESDI. Confidential Enquiry into Stillbirths and Death in Infancy. 5th Annual Report. London, UK: Maternal and Child Health Research Consortium; 1998

6. Hogan MC, Foreman KJ, Naghavi M, et al. Maternal mortality for 181 countries, 1980-2008: a systematic analysis of progress towards Millennium Development Goal 5. Lancet. 2010;375(9726): 1609-1623.

7. McClure JH, Cooper GM, Clutton-Brock TH; Centre for Maternal and Child Enquiries. Saving mothers' lives: reviewing maternal deaths to make motherhood safer: 2006-8: a review. Br J Anaesth. 2011;107(2): $127-132$.

8. Sibai B, Dekker G, Kupferminc M. Pre-eclampsia. Lancet. 2005; 365(9461):785-799.

9. Duckitt K, Harrington D. Risk factors for pre-eclampsia at antenatal booking: systematic review of controlled studies. BMJ. 2005;330(7491) 565.

10. Pijnenborg R, Vercruysse L, Hanssens M. The uterine spiral arteries in human pregnancy: facts and controversies. Placenta. 2006;27(9-10):939-958.

11. Redman CW, Sargent IL, Staff AC. IFPA Senior Award Lecture: making sense of pre-eclampsia - two placental causes of preeclampsia? Placenta. 2014;35 Suppl:S20-S25.

12. Dekker GA, Sibai BM. Etiology and pathogenesis of preeclampsia: current concepts. Am J Obstet Gynecol. 1998;179(5):1359-1375.

13. Hauth JC, Ewell MG, Levine RJ, et al. Pregnancy outcomes in healthy nulliparas who developed hypertension. Calcium for Preeclampsia Prevention Study Group. Obstet Gynecol. 2000;95(1): 24-28. 
14. Gagnon R. Placental insufficiency and its consequences. Eur J Obstet Gynecol Reprod Biol. 2003;110 Suppl 1:S99-S107.

15. Roberts JM, Hubel CA. Oxidative stress in preeclampsia. Am J Obstet Gynecol. 2004;190(5):1177-1178.

16. Borzychowski AM, Sargent IL, Redman CW. Inflammation and pre-eclampsia. Semin Fetal Neonatal Med. 2006;11(5):309-316.

17. National Institute for Health and Care Excellence (NICE). Hypertension in pregnancy: the management of hypertensive disorders during pregnancy. In: NICE CG 107. Manchester, UK: National Institute for Health and Clinical Excellence; 2010. Available from http://www.nice. org.uk/guidance/cg107/resources/guidance-hypertension-in-pregnancypdf. Accessed February 5, 2015.

18. Duley L, Henderson-Smart DJ, Meher S, King JF. Antiplatelet agents for preventing pre-eclampsia and its complications. Cochrane Database Syst Rev. 2007;(2):CD004659.

19. Dekker GA. Risk factors for preeclampsia. Clin Obstet Gynecol. 1999; 42(3):422-435.

20. Lie RT, Rasmussen S, Brunborg H, Gjessing HK, Lie-Nielsen E, Irgens LM. Fetal and maternal contributions to risk of pre-eclampsia: population based study. BMJ. 1998;316(7141):1343-1347.

21. Backes CH, Markham K, Moorehead P, Cordero L, Nankervis CA, Giannone PJ. Maternal preeclampsia and neonatal outcomes. J Pregnancy. 2011;2011:214365.

22. Friedman SA, Schiff E, Kao L, Sibai BM. Neonatal outcome after preterm delivery for preeclampsia. Am J Obstet Gynecol. 1995;172(6): $1785-1792$

23. Sibai BM, Spinnato JA, Watson DL, Hill GA, Anderson GD. Pregnancy outcome in 303 cases with severe preeclampsia. Obstet Gynecol. 1984;64(3):319-325.

24. Bellamy L, Casas JP, Hingorani AD, Williams DJ. Pre-eclampsia and risk of cardiovascular disease and cancer in later life: systematic review and meta-analysis. BMJ. 2007;335(7627):974.

25. Milne F, Redman C, Walker J, et al. The pre-eclampsia community guideline (PRECOG): how to screen for and detect onset of preeclampsia in the community. BMJ. 2005;330(7491):576-580.

26. Kleinrouweler CE, Wiegerinck MM, Ris-Stalpers C, et al. EBM CONNECT Collaboration. Accuracy of circulating placental growth factor, vascular endothelial growth factor, soluble fms-like tyrosine kinase 1 and soluble endoglin in the prediction of pre-eclampsia: a systematic review and meta-analysis. BJOG. 2012;119(7):778-787.

27. Chappell LC, Duckworth S, Seed PT, et al. Diagnostic accuracy of placental growth factor in women with suspected preeclampsia: a prospective multicenter study. Circulation. 2013;128(19):2121-2131.

28. Powers RW, Jeyabalan A, Clifton RG, et al; Eunice Kennedy Shriver National Institute of Child Health Human Development Maternal-Fetal Medicine Units Network. Soluble fms-Like tyrosine kinase 1 (sFlt1), endoglin and placental growth factor (PlGF) in preeclampsia among high risk pregnancies. PLoS One. 2010;5(10):e13263.

29. Andraweera PH, Dekker GA, Roberts CT. The vascular endothelial growth factor family in adverse pregnancy outcomes. Hum Reprod Update. 2012;18(4):436-457.
30. Zhou Y, McMaster M, Woo K, et al. Vascular endothelial growth factor ligands and receptors that regulate human cytotrophoblast survival are dysregulated in severe preeclampsia and hemolysis, elevated liver enzymes, and low platelets syndrome. Am J Pathol. 2002;160(4): $1405-1423$.

31. McLeod L. How useful is uterine artery Doppler ultrasonography in predicting pre-eclampsia and intrauterine growth restriction? CMAJ. 2008;178(6):727-729.

32. Cnossen JS, Morris RK, ter Riet G, et al. Use of uterine artery Doppler ultrasonography to predict pre-eclampsia and intrauterine growth restriction: a systematic review and bivariable meta-analysis. CMAJ. 2008;178(6):701-711.

33. Kenny LC, Broadhurst DI, Dunn W, et al; Screening for Pregnancy Endpoints Consortium. Robust early pregnancy prediction of later preeclampsia using metabolomic biomarkers. Hypertension. 2010;56(4): 741-749.

34. Navaratnam K, Alfirevic Z, Baker PN, et al. A multi-centre phase IIa clinical study of predictive testing for preeclampsia: improved pregnancy outcomes via early detection (IMPROvED). BMC Pregnancy Childbirth. 2013;13:226.

35. Buhimschi IA, Nayeri UA, Zhao G, et al. Protein misfolding, congophilia, oligomerization, and defective amyloid processing in preeclampsia. Science Transl Med. 2014;6(245):245ra292.

36. North RA, McCowan LM, Dekker GA, et al. Clinical risk prediction for pre-eclampsia in nulliparous women: development of model in international prospective cohort. BMJ. 2011;342:d1875.

37. Kenny LC, Black MA, Poston L, et al. Early pregnancy prediction of preeclampsia in nulliparous women, combining clinical risk and biomarkers: the Screening for Pregnancy Endpoints (SCOPE) international cohort study. Hypertension. 2014;64(3):644-652.

38. Myers JE, Baker PN. Hypertensive diseases and eclampsia. Curr Opin Obstet Gynecol. 2002;14(2):119-125.

39. CLASP: a randomised trial of low-dose aspirin for the prevention and treatment of pre-eclampsia among 9364 pregnant women. CLASP (Collaborative Low-dose Aspirin Study in Pregnancy) Collaborative Group. Lancet. 1994;343(8898):619-629.

40. Hofmeyr GJ, Lawrie TA, Atallah AN, Duley L, Torloni MR. Calcium supplementation during pregnancy for preventing hypertensive disorders and related problems. Cochrane Database Syst Rev. 2014;6: CD001059.

41. Altman D, Carroli G, Duley L, et al; Magpie Trial Collaboration Group. Do women with pre-eclampsia, and their babies, benefit from magnesium sulphate? The Magpie Trial: a randomised placebo-controlled trial. Lancet. 2002;359(9321):1877-1890.

42. Koopmans CM, Bijlenga D, Groen H, et al; HYPITAT study group. Induction of labour versus expectant monitoring for gestational hypertension or mild pre-eclampsia after 36 weeks' gestation (HYPITAT): a multicentre, open-label randomised controlled trial. Lancet. 2009;374(9694):979-988
Integrated Blood Pressure Control

\section{Publish your work in this journal}

Integrated Blood Pressure Control is an international, peer-reviewed open-access journal focusing on the integrated approach to managing hypertension and risk reduction. Treating the patient and comorbidities together with diet and lifestyle modification and optimizing healthcare resources through a multidisciplinary team approach constitute key

\section{Dovepress}

features of the journal. This journal is indexed on American Chemical Society's Chemical Abstracts Service (CAS). The manuscript management system is completely online and includes a very quick and fair peerreview system, which is all easy to use. Visit http://www.dovepress.com/ testimonials.php to read real quotes from published authors. 